\title{
Hierarchy of Critical Exponents on Sierpinski fractal resistor networks
}

\author{
M. A. Jafarizadeh ${ }^{a, b, c} *$ M.Mirzaee ${ }^{a, b \dagger}$ and H. Aghlara ${ }^{a, c \ddagger}$ \\ ${ }^{a}$ Department of Theoretical Physics and Astrophysics, Tabriz University, Tabriz 51664, Iran. \\ ${ }^{b}$ Institute for Studies in Theoretical Physics and Mathematics, Tehran 19395-1795, Iran. \\ ${ }^{c}$ Pure and Applied Science Research Center, Tabriz 51664, Iran.
}

November 16, 2018

\begin{abstract}
Using the $S_{3}$-symmetry of Sierpinski fractal resistor networks we determine the current distribution as well as the multifractals spectrum of moments of current distribution by using the real space renormalization group technique based on $([q / 4]+1)$ independent Schure's invariant polynomials of inwards flowing currents.
\end{abstract}

Keywords: Renormalization Group, Fractal, multifractals, Resistor Network . PACs Index: 64.60.AK and 05.50

*E-mail: jafarzadeh@ark.tabrizu.ac.ir

${ }^{\dagger}$ E-mail:mirzaee@ark.tabrizu.ac.ir

${ }^{\ddagger}$ E-mail:aghlara@ark.tabrizu.ac.ir 


\section{INTRODUCTION}

The study of infinite sets of exponents which originated in the field of turbulence [1], has recently become the focus of attention in a number of fields involving fractals or scaling objects [2, 3], ranging from random resistor networks [4, 5, 6], dynamical systems, diffusion limited aggregates (DLA) [7], to localization. What is common to all of these different fields is that, one wants to characterize the properties of a "weight" or "measure" associated to different parts of a fractal object. Modelization of electrical transport properties for inhomogeneous and composite materials by random resistor networks have been the subject of many recent works, Also other physical phenomena such as diffusion problems can be formulated in terms of electrical problem. Distribution of currents (or voltage drops) on a percolating structure in the scaling region are multifractals, in the sense that different moments scale with different exponents, that is, if we consider a system of length $L$, then the $q$-moment of the current distribution :

$$
M_{q}=\sum_{r} I_{r}^{q}
$$

scales as $L^{-D_{q}}$, where $D_{q}$ is by no means a simple function of $q$. Thus each moment scales with its own anomalous dimension. This phenomena is characteristic of multifractals distributions. Actually this set of exponents first appeared in the field of turbulence and has recently become focus of attention in a number different fields such as diffusion limited aggregation, dynamical system and random resistor networks as mentioned above. Here in this paper we study the multifractals structure of current distribution on Sierpinsky fractal, since as Kirkpatric had suggested, the so called back-bone of the percolating random resistor networks could be modeled by a fractal structure and among the fractal objects, the $n$ simplex one is simplest to study the various physical problems from random walk [8, 9, 10, 11] to electrical one on it [5, 6, 12, 13].

Here by using the $S_{3}$-symmetry of Sierpinski fractal resistor networks (see Fig. 1) together 
with the minimization of the electrical power, we have been able to determine the current distribution in Sierpinsky fractals with decimation numbers $b=2,3,4$, and 5 . Then, using the independent Shure's $S_{3}$ invariant polynomials, which is proved that the required number of independent Shure's $S_{3}$-invariant polynomials of degree $q$ is $[q / 4]+1$, with [ ] indicating the greatest integer parts, we have derived the results of reference for $b=2$ up to $q=12$ and we have calculated $D_{q}$ up to $q=22$ for $b=2,3,4$ and 5 . The organization of the article is as follows:

In section 2, we give a brief description of Sierpinski fractals, then in section 3, using the $S_{3}$-symmetry of Sierpinsky fractal resistor networks and minimization of electrical power we have determined the inward flowing current of subfractals. In section 4 we talk about the independent Shure,s $S_{3}$-symmetry invariant polynomials of input currents. Section 5 is about the moments current distributions and their multifractals spectrum where it contains the main results of this paper. The paper ends with a brief conclusion and 5 appendices.

\section{$2 \quad$ Sierpinski Fractal}

To obtain Sierpinski fractal with decimation number $b$, we choose a triangle and divide its sides into $b$ parts and then draw all possible lines through the dividend points parallel to the side of the triangle. Next, having omitted every other inner triangle, we repeat this for the remaining triangles or for the subfractals of the next higher order. This way Sierpinski fractals are constructed. To calculate the fractal dimension, we label subfractals of order $(l+1)$ in terms of partition of $(b-1)$ into 3 positive integers $\lambda_{1}, \lambda_{2}$ and $\lambda_{3}$. Each partition represents a subfractal of order $l$ and $\lambda$ shows the distance of the corresponding subfractal 
from the sides of triangle. As an illustrating example, we show in Figure 2 the method of labeling a Sierpinski fractal with decimation number $b=3$. Obviously, the number of all possible partitions is equal to the distribution of $(b-1)$ objects among three boxes, which is the same as the Bose-Einstein distribution of $(b-1)$ identical bosons in 3 quantum states. This is equal to

$$
c=\frac{(b+1) !}{(b-1) ! 2 !} .
$$

According to the following definition, the fractal dimension $d_{F}$ of a self-similar object is

$$
\left(N^{r}\right)^{d_{F}}=1
$$

with $N$ as the number of similar objects, up to translation and rotation. For self-similar fractals, $N$ is equal to the number of subfractals. Therefore, we have $N=C^{l}$ and $r=b^{-l}$. Hence $d_{F}=\frac{\ln C}{\ln b}$, or

$$
d_{F}=\frac{\ln (b+1) ! /(b-1) ! 2 !}{\ln b} .
$$

\section{Determination of inward flowing current of subfrac- tals}

We denote the $\mathrm{j}$-th inward flowing current of subfractal which corresponds to the partition $\lambda_{1}, \lambda_{2}$ and $\lambda_{3}$ by $I_{\lambda_{1}, \lambda_{2}, \lambda_{3}}\left(\lambda_{1}+\delta_{1, j}, \lambda_{2}+\delta_{2, j}, \lambda_{3}+\delta_{3, j}\right)$. Therefore, $I_{1}, I_{2}$ and $I_{3}$ can be denoted by

$$
I_{b-1,0,0}(b, 0,0), \quad I_{0, b-1,0}(0, b, 0) \quad \text { and } \quad I_{0,0, b-1}(0,0, b) \text {. }
$$


In order to determine the inward flowing currents in terms of $I_{j}, j=1,2$ and 3 , besides using Kirchhoff's law at each node, we have to minimize the power of Sierpinski fractal, that is we minimize the following expression:

$$
\begin{gathered}
\sum_{\text {over possible }} \sum_{\text {partitions }} \sum_{j=1}^{3} I_{\lambda_{1}, \lambda_{2} \lambda_{3}}^{2}\left(\lambda_{1}+\delta_{1, j}, \lambda_{2}+\delta_{2, j}, \lambda_{3}+\delta_{3, j}\right) \\
+\mu_{\lambda_{1}, \lambda_{2}, \lambda_{3}}\left(\sum_{j=1}^{3} I_{\lambda_{1}, \lambda_{2}, \lambda_{3}}\left(\lambda_{1}+\delta_{1, j}, \lambda_{2}+\delta_{2, j}, \lambda_{3}+\delta_{3, j}\right)\right. \\
+2 \sum_{\text {over all }{ }_{\text {nodes }} \nu_{\eta_{1}, \eta_{2}, \eta_{3}}\left(\sum_{j=1}^{3} I_{\eta_{1}, \eta_{2}, \eta_{3}}\left(\eta_{1}-\delta_{1, j}, \eta_{2}-\delta_{2, j}, \eta_{3}-\delta_{3, j}\right) .\right.}
\end{gathered}
$$

where $\mu_{\lambda_{1}, \lambda_{2}, \lambda_{3}}$ and $\nu_{\text {eta }_{1}, \text { eta }_{2}, \eta_{3}}$ are Lagrange multipliers which are considered because of Kirchhoff,s law on each subfractal, and also each node, respectively. By minimizing the energy given by expression (1-2), we get the following equations for the inner flowing currents:

$$
I_{\lambda_{1}, \lambda_{2}, \lambda_{3}}\left(\lambda_{1}+\delta_{1, j}, \lambda_{2}+\delta_{2, j}, \lambda_{3}+\delta_{3, j}\right)+\mu_{\lambda_{1}, \lambda_{2}, \lambda_{3}}+n u_{\lambda_{1}+\delta_{1, j}, \lambda_{2}+\delta_{2, j}, \lambda_{3}+\delta_{3, j}}=0
$$

together with the Kirchhoff's law for each subfractal and each vertex, respectively:

$$
\begin{gathered}
\sum_{j=1}^{3} I_{\lambda_{1}, \lambda_{2}, \lambda_{3}}\left(\lambda_{1}+\delta_{1, j}, \lambda_{2}+\delta_{2, j}, \lambda_{3}+\delta_{3, j}\right)=0 \\
\sum_{j=1}^{3} I_{\eta_{1}, \eta_{2}, \eta_{3}}\left(\eta_{1}-\delta_{1, j}, \eta_{2}-\delta_{2, j}, \eta_{3}-\delta_{3, j}\right)=0 .
\end{gathered}
$$

Now, using the $S_{3}$-permutation symmetry of Sierpinski fractal we propose the following ansatz for the Lagrange multipliers:

$$
\begin{aligned}
& \mu_{\lambda_{1}, \lambda_{2}, \lambda_{3}}=-\sum_{k=1}^{3} a_{\lambda_{k}} I_{k}, \\
& \nu_{\lambda_{1}, \lambda_{2}, \lambda_{3}}=-\sum_{k=1}^{3} b_{\lambda_{k}} I_{k},
\end{aligned}
$$


where $a_{0}$ is assumed to be zero. Substituting the ansatz (3-5) and (3-6) in equation (3-2), then the inward flowing currents can be given in terms of a's and b,s, respectively.

$$
I_{\lambda_{1}, \lambda_{2}, \lambda_{3}}\left(\eta_{1}, \eta_{2}, \eta_{3}\right)=\sum_{k=1}^{3}\left(a_{\lambda_{k}}+b_{\eta_{k}}\right) I_{k} .
$$

Actually one could write the currents in terms of input ones as in (3-7) simply by using the symmetry of simplex fracal where the minimization of power is not required. Finally the a's and b's themselves can be determined through the equations (3-3) and (3-4). Here we determine the currents for $b=2,3,4$ and 5 , respectively. First for $b=2$ we have

$$
\begin{gathered}
I_{1,0,0}(2,0,0)=I_{1}, \quad I_{0,1,0}(0,2,0)=I_{2} \quad, \quad I_{0,0,1}(0,0,2)=I_{3}, \\
I_{\delta_{1, j}, \delta_{2, j}, \delta_{3, j}}\left(\delta_{1, j}+\delta_{1, k}, \delta_{2, j}+\delta_{2, k} \delta_{3, j}+\delta_{3, k}\right)=a_{1} I_{J}+b_{1} I_{j}+b_{1} I_{k}
\end{gathered}
$$

Using equation (3-4) we obtain:

$$
a_{1}+2 b_{1}=0
$$

and using equation (3-4) we get:

$$
1+2 a_{1}+b_{1}=0
$$

Solving the above equations we get the following result:

$$
I_{\delta_{1, j}, \delta_{2, j}, \delta_{3, j}}\left(\delta_{1 j}+\delta_{1 k}, \delta_{2 j}+\delta_{2 k}, \delta_{3 j}+\delta_{3 k}\right)=\frac{\left(I_{k}-I_{j}\right)}{3} .
$$

Similarly for $b=3$ we have

$$
I_{2,0,0}(3,0,0)=I_{1}, \quad I_{0,2,0}(0,3,0)=I_{2}, \quad I_{0,0,2}(0,0,3)=I_{3},
$$




$$
\begin{gathered}
I_{2 \delta_{1, j}, 2 \delta_{2, j}, 2 \delta_{3, j}}\left(2 \delta_{1, j}+\delta_{1, k}, 2 \delta_{2, j}+\delta_{2, k}, 2 \delta_{3, j}+\delta_{3, k}\right)=a_{2} I_{j}+b_{2} I_{j}+b_{1} I_{k}, \\
I_{\delta_{1, j}+\delta_{1, k}, \delta_{2, j}+\delta_{2, k}, \delta_{3, j}+\delta_{3, k}}\left(2 \delta_{1, j}+\delta_{1, k}, 2 \delta_{2, j}+\delta_{2, k}, 2 \delta_{3, j}+\delta_{3, k}\right)=a_{1} I_{j}+a_{1} I_{k}+b_{2} I_{j}+b_{1} I_{k}, \\
I_{\delta_{1, j}+\delta_{1, k}, \delta_{2, j}+\delta_{2, k}, \delta_{3, j}+\delta_{3, k}}\left(\delta_{1, j}+\delta_{1, k}+\delta_{1, l}, \delta_{2, j}+\delta_{2, k}+\delta_{2, l}, \delta_{3, j}+\delta_{3, k}+\delta_{3, l}\right) \\
=a_{1}\left(I_{j}+I_{k}\right)+b_{1}\left(I_{j}+I_{k}+I_{l}\right) .
\end{gathered}
$$

Using equation (3-3) in subfractal $\left(2 \delta_{1, j}, 2 \delta_{2, j}, 2 \delta_{3, j}\right)$, we get

$$
1+2\left(a_{2}+b_{2}\right)-b_{1}=0
$$

Also using equation (3-3) in subfractal $\left(\delta_{1, j}+\delta_{1, K}, \delta_{2, j}+\delta_{2, K}, \delta_{3, j}+\delta_{3, K}\right)$ we get

$$
3 a_{1}+2 b_{1}+b_{2}=0
$$

Also using equation (3-4) in the vertices we have

$$
\begin{gathered}
a_{1}+a_{2}+2 b_{1}=0, \\
a_{1}+2 b_{1}=0, \\
2 a_{1}+3 b_{1}=0 .
\end{gathered}
$$

After solving the above equations we get the following result for the currents for $b=3$

$$
I_{2 \delta_{1, j}, 2 \delta_{2, j}, 2 \delta_{3, j}}\left(2 \delta_{1, j}+\delta_{1, k}, 2 \delta_{2, j}+\delta_{2, k}, 2 \delta_{3, j}+\delta_{3, k}\right)=-\frac{9}{21} I_{j}+\frac{3}{21} I_{k}
$$




$$
\begin{gathered}
I_{\delta_{1, j}+\delta_{1, k}, \delta_{2, j}+\delta_{2, k}, \delta_{3, j}+\delta_{3, k}}\left(2 \delta_{1, j}+\delta_{1, k}, 2 \delta_{2, j}+\delta_{2, k}, 2 \delta_{3, j}+\delta_{3, k}\right) \\
=\frac{9}{21} I_{j}-\frac{3}{21} I_{k}, \\
I_{\delta_{1, j}+\delta_{1, k}, \delta_{2, j}+\delta_{2, k}, \delta_{3, j}+\delta_{3, k}}\left(\delta_{1, j}+\delta_{1, k}+\delta_{1, l}, \delta_{2, j}+\delta_{2, k}+\delta_{2, l}, \delta_{3, j}+\delta_{3, k}+\delta_{3, l}\right) \\
=-\frac{6}{21}\left(I_{j}+I_{k}\right)+\frac{4}{21}\left(I_{j}+I_{k}+I_{l}\right) .
\end{gathered}
$$

By the same procedure explained above, we can calculate the inner inward flowing currents for decimation number $b=4$ and $b=5$, where we quote only the results below and give the details of calculation in Appendix I and II.

\subsection{Inner inward flowing currents corresponding to $b=4$ :}

$$
\begin{gathered}
I_{3 \delta_{1, j}, 3 \delta_{2, j}, 3 \delta_{3, j}}\left(3 \delta_{1, j}+\delta_{1, k}, 3 \delta_{2, j}+\delta_{2, k}, 3 \delta_{3, j}+\delta_{3, k}\right)=-\frac{19}{41} I_{j}+\frac{3}{41} I_{k} \\
I_{2 \delta_{1, j}+\delta_{1, k}, 2 \delta_{2, j}+\delta_{2, k}, 2 \delta_{3, j}+\delta_{3, k}}\left(3 \delta_{1, j}+\delta_{1, k}, \delta_{2, j}+\delta_{2, k}, 3 \delta_{3, j}+\delta_{3, k}\right)=\frac{19}{41} I_{j}-\frac{3}{41} I_{k}, \\
I_{2 \delta_{1, j}+\delta_{1, k}, 2 \delta_{2, j}+\delta_{2, k}, 2 \delta_{3, j}+\delta_{3, k}}\left(2 \delta_{1, j}+2 \delta_{1, k}, 2 \delta_{2, j}+2 \delta_{2, k}, 2 \delta_{3, j}+2 \delta_{3, k}\right) \\
=-\frac{9}{41}\left(I_{j}-I_{k}\right), \\
I_{2 \delta_{1, j}+\delta_{1, k}, 2 \delta_{2, j}+\delta_{2, k}, 2 \delta_{3, j}+\delta_{3, k}}\left(2 \delta_{1, j}+\delta_{1, k}+\delta_{1, l}, 2 \delta_{2, j}+\delta_{2, k}+\delta_{2, l}, 2 \delta_{3, j}+\delta_{3, k}+\delta_{3, l}\right) \\
=-\frac{184}{1353} I_{j}-\frac{52}{1353} I_{k}+\frac{146}{1353} I_{l} \\
I_{\delta_{1, j}+\delta_{1, k}+\delta_{1, l}, \delta_{2, j}+\delta_{2, k}+\delta_{2, l}, \delta_{3, j}+\delta_{3, k}+\delta_{3, l}}\left(2 \delta_{1, j}+\delta_{1, k}+\delta_{1, l}, 2 \delta_{2, j}+\delta_{2, k}+\delta_{2, l}, 2 \delta_{3, j}+\delta_{3, k}+\delta_{3, l}\right)
\end{gathered}
$$




$$
=\frac{368}{1353} I_{j}-\frac{94}{1353}\left(I_{k}+I_{l}\right) .
$$

\subsection{Inner inward flowing currents corresponding to $b=5$ :}

$$
\begin{gathered}
I_{4 \delta_{1, j}, 4 \delta_{2, j}, 4 \delta_{3, j}}\left(4 \delta_{1, j}+\delta_{1, k}, 4 \delta_{2, j}+\delta_{2, k}, 4 \delta_{3, j}+\delta_{3, k}\right)=\frac{283}{591} I_{j}-\frac{41375}{1015929} I_{k}, \\
I_{3 \delta_{1, j}+\delta_{1, k}, 3 \delta_{2, j}+\delta_{2, k}, 3 \delta_{3, j}+\delta_{3, k}}\left(4 \delta_{1, j}+\delta_{1, k}, 4 \delta_{2, j}+\delta_{2, k}, 4 \delta_{3, j}+\delta_{3, k}\right) \\
=-\frac{283}{591} I_{j}+\frac{41375}{1015929} I_{k}, \\
I_{3 \delta_{1, j}+\delta_{1, k}, 3 \delta_{2, j}+\delta_{2, k}, 3 \delta_{3, j}+\delta_{3, k}}\left(3 \delta_{1, j}+2 \delta_{1, k}, 3 \delta_{2, j}+2 \delta_{2, k}, 3 \delta_{3, j}+2 \delta_{3, k}\right) \\
=\frac{51}{197} I_{j}-\frac{25}{197} I_{k}, \\
I_{3 \delta_{1, j}+\delta_{1, k}, 3 \delta_{2, j}+\delta_{2, k}, 3 \delta_{3, j}+\delta_{3, k}}\left(3 \delta_{1, j}+\delta_{1, k}+\delta_{1, l}, 3 \delta_{2, j}+\delta_{2, k}+\delta_{2, l}, 3 \delta_{3, j}+\delta_{3, k}+\delta_{3, l}\right) \\
=2 \frac{17486}{112881} I_{j}+2 \frac{2206}{112881} I_{k}-2 \frac{2448}{37627} I_{l}, \\
I_{2 \delta_{1, j}+2 \delta_{1, k}, 2 \delta_{2, j}+2 \delta_{2, k}, 2 \delta_{3, j}+2 \delta_{3, k}}\left(3 \delta_{1, j}+2 \delta_{1, k}, 3 \delta_{2, j}+2 \delta_{2, k}, 3 \delta_{3, j}+2 \delta_{3, k}\right) \\
=-\frac{51}{197} I_{j}-\frac{25}{197} I_{k}, \\
I_{2 \delta_{1, j}+\delta_{1, k}+\delta_{1, l}, 2 \delta_{2, j}+\delta_{2, k}+\delta_{2, l}, 2 \delta_{3, j}+\delta_{3, k}+\delta_{3, l}}\left(2 \delta_{1, j}+2 \delta_{1, k}+\delta_{1, l}, 2 \delta_{2, j}+2 \delta_{2, k}+\delta_{2, l}, 2 \delta_{3, j}+2 \delta_{3, k}+\delta_{3, l}\right) \\
I_{2 \delta_{1, j}+2 \delta_{1, k}, 2 \delta_{2, j}+2 \delta_{2, k}, 2 \delta_{3, j}+2 \delta_{3, k}+\delta_{1, k}, 2 \delta_{2, j}+\delta_{2, k}+\delta_{2, l}, 2 \delta_{3, j}+\delta_{3, k}+\delta_{3, l}}\left(2 \delta_{1, j}+2 \delta_{1, k}+\delta_{1, l}, 2 \delta_{2, j}+2 \delta_{2, k}+\delta_{2, l}, 2 \delta_{3, j}+2 \delta_{3, k} \delta_{3, l}\right) \\
=2 \frac{5138}{112881}\left(I_{k}+I_{l}\right)-4 \frac{34972}{112881} I_{j}, \\
\left.=\delta_{1, l}, 3 \delta_{2, j}+\delta_{2, k}+\delta_{2, l}, 3 \delta_{3, j}+\delta_{3, k}+\delta_{3, l}\right) \\
=
\end{gathered}
$$




$$
=2 \frac{18847}{3047787} I_{j}-2 \frac{14356}{3047787} I_{k}+\frac{624}{3047787} I_{l} .
$$

\section{Shure's Polynomials of Inward Flowing Currents}

Shure's $S_{3}$-invariant polynomials are homogeneous polynomials of degree 3 of variables $I_{1}, I_{2}$ and $I_{3}$ :

$$
s_{\lambda_{1}, \lambda_{2}, \lambda_{3}}=\sum_{\text {permutation }} I_{\text {of }(1,2,3)} I_{1}^{\lambda_{1}} I_{2}^{\lambda_{2}} I_{3}^{\lambda_{3}}
$$

where $\lambda_{1}, \lambda_{2}, \lambda_{3}$ are partitions of $\mathrm{m}$ into 3 non-negative integers, that is:

$$
\lambda_{1}+\lambda_{2}+\lambda_{3}=m .
$$

Because of the following equation due to Kirchhoff's law:

$$
S_{1}=\sum_{k=1}^{3} I_{k}=0,
$$

all Schure's polynomials of degree $\mathrm{m}$, corresponding to all possible partitions of $\mathrm{m}$, are not independent. In calculation of the multifractals critical exponents $D_{q}$, we must use the independent ones. By multiplying both sides of (4-2) by $S_{\lambda_{1}, \lambda_{2}, \lambda_{3}}$, we get

$$
0=S_{1} S_{\lambda_{1}, \lambda_{2}, \lambda_{3}}=\sum a_{\mu_{1}, \mu_{2}, \mu_{3}} S_{\mu_{1}, \mu_{2}, \mu_{3}},
$$

where $\left(\mu_{1}, \mu_{2}, \mu_{3}\right)$ and $\left(\lambda_{1}, \lambda_{2}, \lambda_{3}\right)$ correspond to partition of $m-1$ and $m$ respectively. From the formula $(2-10)$ it follows that there are $P_{3}(m+1)$ constraint over $P_{3}(m)$ shure polynomials of degree $\mathrm{m}$, where $p_{3}(m)$ takes all possible partitions of $m$ into 3 non-negative integers. Therefore, the number of invariant polynomials of degree $\mathrm{m}$ is: 


$$
P_{3}(m)-P_{3}(m-1)
$$

For example for $m=2$ we have

$$
0=S_{1} S_{1}=S_{2}+2 S_{1,1}
$$

therefore, using the above equation we can write $S_{1,1}$ in terms of $S_{2}$ as:

$$
S_{1,1}=-\frac{S_{2}}{2}
$$

Thus we have only one invariant polynomial for $q=2$. Also in the case of $q=4$ we have

$$
\begin{gathered}
S_{1} S_{3}=S_{4}+S_{3,1}=0, \\
S_{1} S_{2,1}=S_{3,1}+2 S_{2,2}+S_{2,1,1}=0, \\
S_{1} S_{1,1,1}=S_{2,1,1}=0,
\end{gathered}
$$

hence there is only one independent polynomial such as $S_{4}$ and the others can be written in terms of $S_{4}$ as follows:

$$
\begin{gathered}
S_{3,1}=-S_{4}, \quad S_{2,2}=\frac{S_{4}}{2} \\
S_{2,1,1}=0
\end{gathered}
$$

For $q=6$ we have:

$$
\begin{gathered}
S_{1} S_{5}=S_{6}+S_{5,1}=0 \\
S_{1} S_{4,1}=S_{5,1}+S_{4,2}+S_{4,1,1}=0 \\
S_{1} S_{3,2}=S_{4,2}+2 S_{3,3}+S_{3,2,1}=0 \\
S_{1} S_{3,1,1}=S_{4,1,1}+S_{3,2,1}=0 .
\end{gathered}
$$


Therefore, there are only two independent invariant polynomials such as $S_{6}, S_{3,3}$ and the other dependent one can be written in terms of them as follows:

$$
S_{5,1}=-S_{6}, \quad S_{4,2}=\frac{S_{6}}{2}-S_{3,3}, \quad S_{321}=-\frac{S_{6}}{2}-S_{3,3}, \quad S_{4,1,1}=\frac{S_{6}}{2}+S_{3,3}
$$

In Appendix III, we have proved that the number of independent Schure's invariant polynomials of degree $q$ is equal to:

$$
[q / 4]+1
$$

where [ ] means the greatest integer part.

Below we give some of the constraints over Schure's invariant polynomials of degrees $q=8$ and 10 which are occurring through imposing the Kirschhof's law over Schure's polynomials of order eight:

$$
\begin{gathered}
S_{8}+S_{7,1}=0, \quad S_{7,1}+S_{6,2}+2 S_{6,1,1}=0, \\
S_{6,2}+S_{5,3}+S_{5,2,1}=0, \quad S_{6,1,1}+S_{5,2,1}=0, \\
S_{5,3}+2 S_{4,4}+S_{4,3,1}=0, \quad S_{5,2,1}+S_{4,3,1}+2 S_{4,2,2}=0,
\end{gathered}
$$

where, $S_{8}$ and $S_{4,2,2}$ are considered as the invariant polynomials and other dependent invariant can be expressed in terms of them as follows:

$$
\begin{gathered}
S_{7,1}=-S_{8}, \quad S_{3,3,2}=S_{4,2,2}, \\
S_{4,3,1}=-S_{4,2,2}, \quad S_{5,2,1}=5 S_{4,2,2}, \\
S_{6,1,1}=-5 S_{4,2,2}, \quad S_{6,2}=S_{8}+10 S_{4,2,2}, \\
S_{5,3}=-15 S_{4,2,2}-S_{8}, \quad S_{4,4}=\frac{S_{8}+16 S_{4,2,2}}{2} .
\end{gathered}
$$

Constrains over Schure's polynomials of order ten are: 


$$
\begin{gathered}
S_{10}+S_{9,1}=0, \quad S_{8,2}+S_{9,1}+2 S_{8,1,1}=0 \\
S_{8,2}+S_{7,3}+S_{7,2,1}=0, \quad S_{8,1,1}+S_{7,2,1}=0 \\
S_{7,3}+S_{6,4}+S_{6,3,1}=0, \quad S_{7,2,1}+S_{6,3,1}+2 S_{6,2,2}, \\
S_{6,4}+2 S_{5,5}+S_{5,4,1}=0 \quad S_{6,3,1}+S_{5,4,1}+S_{5,3,2}=0 \\
S_{6,2,2}+S_{5,3,2}=0
\end{gathered}
$$

where $S_{10}$ and $S_{4,4,2}$ are considered as the invariant polynomials and other dependent invariants can be expressed in terms of them as follows:

$$
\begin{gathered}
S_{4,3,3}=0, \quad S_{5,3,2}=-2 S_{4,4,2}, \\
S_{6,2,2}=2 S_{4,4,2}, \quad S_{9,1}=-S_{10} \\
S_{5,4,1}=-S_{4,4,2}, \quad S_{6,3,1}=3 S_{4,4,2}, \\
S_{7,2,1}=-7 S_{4,4,2}, \quad S_{8,1,1}=7 S_{4.4,2}, \\
S_{8,2}=S_{10}-14 S_{4,4,2}, \quad S_{7,3}=21 S_{4,4,2}-S_{10} \\
S_{6,4}=S_{10}-24 S_{4,4,2}, \quad S_{5,5}=\frac{25 S_{4,4,2}-S_{10}}{2},
\end{gathered}
$$

In Appendix IV we use the constraints concerned with the invariant polynomials of order up to 22 to express the dependent invariant polynomials in terms of the independent ones. 


\section{Moments of Current Distribution and Multifractal Spectrum}

In order to study the multifractals behaviour of current distribution we consider their $q$ moments defined as:

$$
M_{q}(n)=\sum_{r} I_{r}(n)^{q}
$$

where $I_{r}$ is the current in the $r$-th bond of subfractals of generation level n. From the $S_{3}$ symmetry of Sierpinsky fractal, it is clear that the $q$-moments depend only on the independent Schure's $S_{3}$ invariant polynomials of degree q of input currents defined in section IV, that is

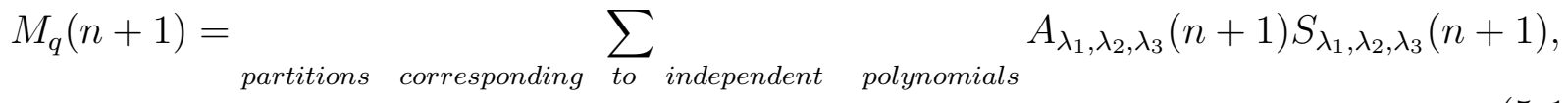

where $A_{\lambda_{1}, \lambda_{2}, \lambda_{3}}$,S are some constants.

On the other hand, $M_{q}(n+1)$ can be written in terms of the invariant polynomials of their level $n$ subfractals, that is

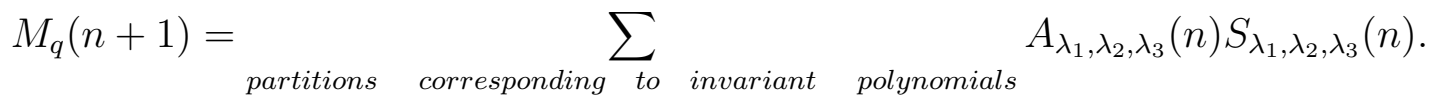

By comparing the expressions (5-1) and (5-2) we obtain the recursion relations between $A_{\lambda_{1}, \lambda_{2}, \lambda_{3}}(n)$ and $A_{\lambda_{1}, \lambda_{2}, \lambda_{3}}(n+1)$. Then the scaling factor is defined as:

$$
\lambda(q)=\lim _{n \longrightarrow \infty} \frac{M_{q}(n+1)}{M_{q}(n)} .
$$

Obviously $\lambda(q)$ is the maximum eigenvalue of the matrix connecting $A(n)$ and $A(n+1)$. Then $D(q)$, the multifractals scaling exponents, are defined as: 


$$
D(q)=\frac{\ln (\lambda(q))}{\ln (b)}
$$

since the $M_{q}(n)$ scale as:

$$
\operatorname{Lim}_{n \longrightarrow \infty} M_{q}(n)=L_{n}^{D(q)}
$$

where $L_{n}=b^{n}$.

Now, as an example, we obtain $D_{2}$, the power scaling exponent of Sierpinsky fractal with decimation numbers $b=2,3,4,4$ and 5 .

According to formula (4-4) for $q=2$ we have only one independent invariant polynomial, where we can consider $S_{2}$ as the independent invariant polynomial. Therefore the total power is proportional to $S_{2}$, that is:

$$
P(n+1)=A_{2}(n+1) S_{2}(n+1) .
$$

It is straightforward to show that:

$$
\begin{gathered}
\frac{A_{2}(n+1)}{A_{2}(n)}=\frac{5}{3} \quad \text { for } \quad b=2 \\
\frac{A_{2}(n+1)}{A_{2}(n)}=\frac{45}{21} \quad \text { for } \quad b=3 \\
\frac{A_{2}(n+1)}{A_{2}(n)}=\frac{3399}{1353} \quad \text { for } \quad b=4 \\
\frac{A_{2}(n+1)}{A_{2}(n)}=\frac{8576091}{3047787} \quad \text { for } \quad b=5
\end{gathered}
$$

Therefore, we have:

$$
\begin{array}{ll}
D(2)=.7369655945 & \text { for } b=2 \\
D(2)=.6937297714 & \text { for } b=3 \\
D(2)=.6644742613 & \text { for } b=4 \\
D(2)=.6428097998 & \text { for } b=5
\end{array}
$$


In case of $D(4)$,we have to consider the $S_{4}, S_{3,1}, S_{2,2}, S_{2,1,1}$, and due to relations (4-5) only one of them, say $S_{4}$, is independent and the others can be written in terms of it. Again by computing the fourth moments of currents of fractals which are proportional to $S_{4}$ :

$$
M_{4}(n)=A_{4}(n) S_{4}(n) \quad M_{4}(n+1)=A_{4}(n+1) S_{4}(n+1)
$$

one can easily show that:

$$
\begin{array}{ll}
\frac{A_{4(n+1)}}{A_{4(n)}}=1.222222222 & \text { for } \quad b=2 \\
\frac{A_{4(n+1)}}{A_{4(n)}}=1.288213244 & \text { for } \quad b=3 \\
\frac{A_{4(n+1)}}{A_{4(n)}}=1.323683604 & \text { for } \quad b=4 \\
\frac{A_{4(n+1)}}{A_{4(n)}}=1.231193828 & \text { for } \quad b=5 .
\end{array}
$$

Hence using the formula (5-4) we get

$$
\begin{array}{ll}
D(4)=.2895066169 & \text { for } b=2 \\
D(4)=.2305237058 & \text { for } b=3 \\
D(4)=.2022791602 & \text { for } b=4 \\
D(4)=.1292279056 & \text { for } b=5
\end{array}
$$

With the above explained prescription, we can calculate the higher moments and consequently higher multifractals exponents, where we quote only the multifractals exponents below in the remaining part of this section and give the other information such as the recursion relations in appendix $\mathrm{V}$ : 
Table 1: The multifractals scaling exponents for $b=2,3,4,5$ and $q=$ $6,8,10,12,14,16,18,20,22$

\begin{tabular}{|c|c|c|c|c|}
\hline & $\mathrm{b}=2$ & $\mathrm{~b}=3$ & $\mathrm{~b}=4$ & $\mathrm{~b}=5$ \\
\hline$D(q=6)$ & 0.08779681671 & .06123675596 & .04899319186 & .02514677379 \\
\hline$D(q=8)$ & .02283703573 & .01502761610 & .01395480248 & .006252791011 \\
\hline$D(q=10)$ & .005703291923 & .003671203776 & .002879616683 & .001058598646 \\
\hline$D(q=12)$ & .001418367685 & .0009335494363 & .0007112996346 & .0002227018237 \\
\hline$D(q=14)$ & .0003533794924 & .0002242180076 & .0001768548943 & .00006087603594 \\
\hline$D(q=16)$ & .00008819069499 & .00005581142757 & .00004410760965 & .00001057440927 \\
\hline$D(q=18)$ & .00002202978507 & .00001392382295 & .00001101561388 & .000001496172722 \\
\hline$D(q=20)$ & .000005503871083 & .000003476196968 & .000002732459233 & .00000008760821964 \\
\hline$D(q=22)$ & .000001376330413 & .000002165456544 & .0000006881652063 & .00000005343480209 \\
\hline
\end{tabular}

Using the above results, the best fit we can get for various multifractals exponents are:

$$
\begin{gathered}
D(q, b=2)=1+4 \times 2^{-q}, \\
D(q, b=3)=1+51.47353178 \times 3^{-q}, \\
D(q, b=4)=1+291.7913871 \times 4^{-q}, \\
D(q, b=5)=1+650.6706017 \times 5^{-q},
\end{gathered}
$$

where the first formula is the same as the formula of reference [5]. The above formulas show the scaling behaviour of the multifractals spectra.

\section{Appendix I: Calculation of currents of $b=4$.}

Here in this Appendix we give the detail of the calculation of inner inward flowing currents corresponding to decimation number $\mathbf{b}=\mathbf{4}$. Following the procedure of section III, for $\mathbf{b}=\mathbf{4}$ 
we have:

$$
I_{3 \delta_{1, j}, 3 \delta_{2, j}, 3 \delta_{3, j}}\left(4 \delta_{1, j}, 4 \delta_{2, j}, 4 \delta_{3, j}\right)=I_{j},
$$

$$
I_{3 \delta_{1, j}, 3 \delta_{2, j}, 3 \delta_{3, j}}\left(3 \delta_{1, j}+\delta_{1, k}, 3 \delta_{2, j}+\delta_{2, k}, 3 \delta_{3, j}+\delta_{3, k}\right)=a_{3}(3) I_{j}+b_{31}(3) I_{j}+b_{31}(1) I_{k},
$$

$I_{2 \delta_{1, j}+\delta_{1, k}, 2 \delta_{2, j}+\delta_{2, k}, 2 \delta_{3, j}+\delta_{3, k}}\left(3 \delta_{1, j}+\delta_{1, k}, 3 \delta_{2, j}+\delta_{2, k}, 3 \delta_{3, j}+\delta_{3, k}\right)=a_{21}(2) I_{j}+a_{21}(1) I_{k}+b_{31}(3) I_{j}+b_{31}(1) I_{k}$,

$I_{2 \delta_{1, j}+\delta_{1, k}, 2 \delta_{2, j}+\delta_{2, k}, 2 \delta_{3, j}+\delta_{3, k}}\left(2 \delta_{1, j}+2 \delta_{1, k}, 2 \delta_{2, j}+2 \delta_{2, k}, 2 \delta_{3, j}+2 \delta_{3, k}\right)=a_{21}(2) I_{j}+a_{21}(1) I_{k}+b_{22}(2)\left(I_{j}+I_{k}\right)$,

$$
\begin{gathered}
I_{2 \delta_{1, j}+\delta_{1, k}, 2 \delta_{2, j}+\delta_{2, k}, 2 \delta_{3, j}+\delta_{3, k}}\left(2 \delta_{1, j}+\delta_{1, k}+\delta_{1, l}, 2 \delta_{2, j}+\delta_{2, k}+\delta_{2, l}, 2 \delta_{3, j}+\delta_{3, k}+\delta_{3, l}\right) \\
=a_{21}(2) I_{j}+a_{21}(1)+b_{211}(2) I_{j}+b_{211}(1)\left(I_{k}+I_{l}\right)
\end{gathered}
$$

$$
\begin{gathered}
I_{\delta_{1, j}+\delta_{1, k}+\delta_{1, l}, \delta_{2, j}+\delta_{2, k}+\delta_{2, l}, \delta_{3, j}+\delta_{3, k}+\delta_{3, l}}\left(2 \delta_{1, j}+\delta_{1, k}+\delta_{1, l}, 2 \delta_{2, j}+\delta_{2, k}+\delta_{2, l}, 2 \delta_{3, j}+\delta_{3, k}+\delta_{3, l}\right) \\
=-47 a_{111}(1)\left(I_{j}+I_{k}+I_{l}\right)+b_{211}(2) I_{j}+b_{211}(1)\left(I_{k}+I_{l}\right) .
\end{gathered}
$$

Now, imposing Kirchhoff's law on subfractals and on vertices, we get the following equations for $\mathbf{a}$ and $\mathbf{b}$

$$
\begin{aligned}
& 1+2 a_{3}(3)+b_{31}(3)-b_{31}(1)=0, \\
& 3 a_{21}(2)+b_{31}(3)+b_{22}(2)+2 b_{211}(2)-b_{211}(1)=0
\end{aligned}
$$




$$
\begin{aligned}
& 3 a_{21}(2)+b_{31}(1)+b_{22}(2)+b_{211}(1)=0 \\
& 3 a_{111}(2)+b_{211}(2)=0 \\
& a_{21}(1)+2 b_{31}(1)=0 \\
& a_{3}(3)+b_{21}(2)+2 b_{31}(3)=0 \\
& a_{21}(2)+a_{21}(1)+2 b_{22}(2)=0 \\
& 2 a_{21}(2)+a_{111}(1)+3 b_{211}(2)=0 \\
& a_{21}(1)+a_{111}(1)+3 b_{211}(1)=0 \\
& 3 a_{111}(1)+4 b_{1111}(1)=0
\end{aligned}
$$

By solving the above equations we can determine inner inward flowing currents corresponding to decimation number $\mathbf{b}=\mathbf{4}$ which is given in subsection 3.1.

\section{Appendix II: Calculation of currents of $\mathbf{b}=\mathbf{5}$.}

Here in this Appendix we give the detail of the calculation of inner inward flowing currents corresponding to decimation number $\mathbf{b}=\mathbf{5}$. Following the procedure of section III, for $\mathbf{b}=\mathbf{5}$ we have:

$$
\begin{gathered}
I_{4 \delta_{1, j}, 4 \delta_{2, j}, 4 \delta_{3, j}}\left(5 \delta_{1, j}, 5 \delta_{2, j}, 5 \delta_{3, j}\right)=I_{j} \\
I_{4 \delta_{1, j}, 4 \delta_{2, j}, 4 \delta_{3, j}}\left(4 \delta_{1, j}+\delta_{1, k}, 4 \delta_{2, j}+\delta_{2, k}, 4 \delta_{3, j}+\delta_{3, k}\right)=a_{4}(4) I_{j}+b_{41}(4) I_{j}+b_{41}(1) I_{k},
\end{gathered}
$$

$I_{3 \delta_{1, j}+\delta_{1, k}, 3 \delta_{2, j}+\delta_{2, k}, 3 \delta_{3, j}+\delta_{3, k}}\left(4 \delta_{1, j}+\delta_{1, k}, 4 \delta_{2, j}+\delta_{2, k}, 4 \delta_{3, j}+\delta_{3, k}\right)=a_{31}(3) I_{j}+a_{31}(1) I_{k}+b_{41}(4) I_{j}+b_{41}(1) I_{k}$, 


$$
\begin{gathered}
I_{3 \delta_{1, j}+\delta_{1, k}, 3 \delta_{2, j}+\delta_{2, k}, 3 \delta_{3, j}+\delta_{3, k}}\left(3 \delta_{1, j}+2 \delta_{1, k}, 3 \delta_{2, j}+2 \delta_{2, k}, 3 \delta_{3, j}+2 \delta_{3, k}\right) \\
=a_{31}(3) I_{j}+a_{31}(1) I_{k}+b_{32}(3) I_{j}+b_{32}(3) I_{k}, \\
I_{3 \delta_{1, j}+\delta_{1, k}, 3 \delta_{2, j}+\delta_{2, k}, 3 \delta_{3, j}+\delta_{3, k}}\left(3 \delta_{1, j}+\delta_{1, k}+\delta_{1, l}, 3 \delta_{2, j}+\delta_{2, k}+\delta_{2, l}, 3 \delta_{3, j}+\delta_{3, k}+\delta_{3, l}\right) \\
=a_{31}(3) I_{j}+a_{31}(1)+b_{311}(3) I_{j}+b_{311}(1)\left(I_{k}+I_{l}\right),
\end{gathered}
$$

$I_{2 \delta_{1, j}+2 \delta_{1, k}, 2 \delta_{2, j}+2 \delta_{2, k}, 2 \delta_{3, j}+2 \delta_{3, k}}\left(3 \delta_{1, j}+2 \delta_{1, k}, 3 \delta_{2, j}+2 \delta_{2, k}, 3 \delta_{3, j}+2 \delta_{3, k}\right)=a_{22}(2)\left(I_{j}+I_{k}\right)+b_{32}(3) I_{j}+b_{32}(2) I_{k}$.

Again imposing Kirchhoff's law on subfractals and on vertices, we get the following equations for $\mathbf{a}$ and $\mathbf{b}$ :

$$
\begin{aligned}
& 1+2 a_{4}(4)+b_{41}(3)-b_{41}(1)=0 \\
& 3 a_{31}(3)+b_{41}(4)+b_{32}(3)+b_{311}(3)-b_{311}(1)=0 \\
& 3 a_{31}(1)+b_{41}(1)+b_{32}(2)=0 \\
& 3 a_{22}(1)+b_{32}(3)+b_{32}(2)+b_{221}(2)-b_{221}(1)=0 \\
& 3 a_{211}(2)+b_{311}(3)+2 b_{221}(2)-b_{2111}(1)=0, \\
& 3 a_{211}(2)+b_{311}(1)+b_{221}(2)+b_{221}(1)=0 \\
& 3 a_{1111}(2)+b_{2111}(2)+3 b_{2111}(1)=0 \\
& a_{4}(4)+a_{31}(3)+2 b_{41}(4)=0 \\
& a_{31}(1)+2 b_{41}(1)=0 \\
& a_{31}(3)+a_{22}(2)+2 b_{32}(3)=0 \\
& a_{31}(1)+a_{22}(2)+2 b_{32}(2)=0 \\
& 2 a_{31}(3)+a_{211}(2)+3 b_{311}(3)=0
\end{aligned}
$$




$$
\begin{aligned}
& a_{31}(1)+a_{211}(1)+3 b_{311}(1)=0, \\
& a_{22}(2)+a_{211}(2)+a_{211}(1)+3 b_{221}(2)=0 \\
& 2 a_{211}(1)+3 b_{221}(1)=0 \\
& 3 a_{211}(2)+a_{1111}(1)+4 b_{2111}(2)=0 \\
& 2 a_{211}(1)+a_{1111}(1)+4 b_{2111}(1)=0 \\
& 4 a_{1111}(1)+5 b_{11111}(1)=0
\end{aligned}
$$

By solving the above equations we can determine inner inward flowing currents corresponding to decimation number $\mathbf{b}=\mathbf{5}$ which is given in subsection 3.2.

Appendix III: Proof of the formula (4-6):

Here we give the proof of the formula (4-6). The number of independent Shure's invariant polynomials of degree $2 k$ of 3 variables $I_{1}, I_{2}, I_{3}$ with the constraint:

$$
I_{1}+I_{2}+I_{3}=0
$$

is equal to

$$
P_{3}(2 K)-P_{3}(2 k-1)
$$

where $P_{3}(m)$ is the number of partition of $m$ into at most three independent non-negative integers. If we define $M_{k}(n)$, the number of partitions of $n$ into exactly $k$ non-negative integers, then we have

$$
P_{3}(n)=\sum_{k=1}^{3} M_{k}(n) .
$$

Obviously

$$
M_{1}(2 k)=M_{1}(2 k-1)
$$

and

$$
M_{2}(2 k)=M_{2}(2 k-1)+1, \quad M_{2}(2 k)=M_{2}(2 k+1) .
$$


If we denote the partition of $2 k, 2 k-1$ and $2 k+1$ into two non-negative integers respectively by: $\left(l_{1}, l_{2}\right),\left(m_{1}, m_{2}\right)$ and $\left(n_{1}, n_{2}\right)$ then in the case of $l_{2}=m_{2}=n_{2}$ we will have

$$
l_{1}=m_{1}-1=n_{1}+1 \text {. }
$$

Therefore, for all values of $l_{1}>k$, there is a one to one correspondence between the $M_{2}(2 k), M_{2}(2 k-1)$ and $M_{2}(2 k+1)$. Only for $l_{1}=k, n_{1}$ can be equal to $k+1$, but $m_{1}$ cannot be equal to $k-1$, thereof the relations (III-4) and (III-5) follows. Now, we are ready to prove that

$$
M_{3}(2 k)=M_{3}(2 k-1)+[k / 3] .
$$

If we denote the partition of $2 k$ and $2 k-1$ into three non-negative integers by $\left(l_{1}, l_{2}, l_{3}\right)$ and $\left(m_{1}, m_{2}, m_{3}\right)$ respectively, then using the relations (III-4) and (III-5), we can prove $M_{3}(2 k)=$ $M_{3}(2 k-1)$ for $m_{1}=l_{1}=o d d$; and for $l_{1}=m_{1}=$ even, we would have $M_{3}(2 k)=M_{3}(2 k-1)$. Since $l_{1}$ takes values between 1 and $[2 k / 3]$, where $[[2 k / 3] / 2]=[k / 3]$ of them correspond to even values of $l_{1}$, the relation (III-7) follows and the proof is complete.

\section{APPENDIX IV:Solution of Constraints Over Schure's invariants polyno-} mials:

Here in this appendix by solving the constraints over Schure's polynomials of degree 12, $14,16,18,20$ and 22 , we have expressed the dependent invariant polynomials in terms of the independent invariant polynomials.

1) Solution of Constraints of degree 12:

The invariant polynomials $S_{12}, S_{8,2,2}$ and $S_{6,3,3}$ are considered to be independent and the other dependent invariant polynomials can be written in terms of them as follows:

$$
\begin{gathered}
S_{5,4,3}=-S_{6,3,3}, \quad S_{4,4,4}=\frac{S_{6,3,3}}{3} \\
S_{7,3,2}=-S_{8,2,2}, \quad S_{6,4,2}=S_{8,2,2}-2 S_{6,3,3},
\end{gathered}
$$




$$
\begin{gathered}
S_{5,5,2}=\frac{3 S_{6,3,3}-S_{8,2,2}}{2}, \quad S_{6,5,1}=\frac{S_{8,2,2}-3 S_{5,3,3}}{2} \\
S_{7,4,1}=\frac{7 S_{6,3,3}-3 S_{8,2,2}}{2}, \quad S_{8,3,1}=\frac{5 S_{8,2,2}-7 S_{6,3,3}}{2}, \\
S_{9,2,1}=\frac{7 S_{6,3,3}-9 S_{8,2,2}}{2}, \quad S_{10,1,1}=\frac{9 S_{8,2,2}-7 S_{6,3,3}}{2}, \\
S_{11,1}=-S_{12}, \\
S_{10,2}=S_{12}+7 S_{6,3,3}-9 S_{8,2,2}, \\
S_{9,3}=\frac{27 S_{8,2,2}-21 S_{6,3,3}-2 S_{12}}{2}, \\
S_{8,4}=\frac{28 S_{6,3,3}-32 S_{8,2,2}+2 S_{12}}{2}, \\
S_{7,5}=\frac{35 S_{8,2,2}-2 S_{12}-35 S_{6,3,3}}{2}, \\
S_{6,6}=\frac{2 S_{12}+38 S_{6,3,3}-36 S_{8,2,2}}{4} .
\end{gathered}
$$

2) Solution of Constraints of degree 14:

The invariant polynomials $S_{14}, S_{6,6,2}$ and $S_{5,5,4}$ are considered to be independent one and the other dependent invariant polynomials can be written in terms of them as follows:

$$
\begin{gathered}
S_{10,3,1}=7 S_{6,2,2}-23 S_{5,5,4}, \\
S_{8,5,1}=3 S_{6,6,2}-S_{5,5,4}, \\
S_{9,4,1}=-5 S_{6,6,2}+7 S_{5,5,4}, \\
S_{8,3,3}=-5 S_{5,5,4}, \quad S_{7,4,3}=5 S_{5,5,4}, \\
S_{6,5,3}=-S_{5,5,4}, \quad S_{6,4,4}=-2 S_{5,5,4}, \\
S_{9,5}=-S_{14}+45 S_{6,6,2}-195 S_{5,5,4}, \\
S_{10,4}=S_{14}-40 S_{6,6,2}+188 S_{5,5,4}, \\
S_{11,3}=-S_{14}+33 S_{6,6,2}-165 S_{5,5,4}, \\
S_{7,7}=\frac{-S_{14}+49 S_{6,6,2}-196 S_{5,5,4},}{2}
\end{gathered}
$$




$$
\begin{gathered}
S_{8,6}=S_{14}-48 S_{6,6,2}+196 S_{5,5,4}, \\
S_{13,1}=-S_{14}, \\
S_{8,4,2}=2 S_{6,62}-6 S_{5,5,4}, \\
S_{12,2}=S_{14}-22 S_{6,6,2}+110 S_{5,5,4}, \\
S_{9,3,2}=-2 S_{6,6,2}+16 S_{5,5,4}, \\
S_{7,5,2}=-2 S_{6,6,2}+S_{5,5,4}, \\
S_{10,2,2}=2 S_{6,6,2}-16 S_{5,5,4}, \\
S_{7,6,1}=-S_{6,6,2}, \\
S_{11,2,1}=-11 S_{6,6,2}+55 S_{5,5,4}, \\
S_{12,1,1}=11 S_{6,6,2}-55 S_{5,5,4} .
\end{gathered}
$$

3) Solution of Constraints of degree 16: The invariant polynomials $S_{16}, S_{7,7,2}, S_{6,6,4}$ are considered to be independent and the other dependent invariant polynomials can be written in terms of them as follows:

$$
\begin{gathered}
S_{6,5,5}=0 \\
S_{7,5,4}=-2 S_{6,6,4}, \quad S_{7,6,3}=-S_{6,6,4}, \\
S_{8,8,4}=2 S_{6,6,4}, \quad S_{8,5,3}=3 S_{6,6,4}, \\
S_{8,7,1}=-S_{7,7,2}, \quad S_{8,6,2}=-2 S_{7,7,2}+S_{6,6,4}, \\
S_{9,4,3}=-7 S_{6,6,4}, \quad S_{9,5,2}=2 S_{7,7,2}-4 S_{6,6,4}, \\
S_{9,6,1}=3 S_{7,7,2}-S_{6,6,4}, \quad S_{10,3,3}=7 S_{6,6,4}, \\
S_{10,4,2}=-2 S_{7,7,2}+11 S_{6,6,4}, \\
S_{10,5,1}=-5 S_{7,7,2}+5 S_{6,6,4}, \\
S_{11,3,2}=2 S_{7,7,2}-25 S_{6,6,4},
\end{gathered}
$$




$$
\begin{gathered}
S_{11,4,1}=7 S_{7,7,2}-16 S_{6,6,4}, \\
S_{12,2,2}=-2 S_{7,7,2}+25 S_{6,6,4}, \\
S_{12,3,1}=-9 S_{7,7,2}+41 S_{6,6,4}, \\
S_{13,2,1}=13 S_{7,7,2}-91 S_{6,6,4}, \\
S_{14,1,1}=-13 S_{7,7,2}+91 S_{6,6,4}, \\
S_{15,1}=-S_{16}, \\
S_{14,2}=S_{16}+26 S_{7,7,2}-182 S_{6,6,4}, \\
S_{13,3}=-S_{16}-39 S_{7,7,2}+273 S_{6,6,4}, \\
S_{12,4}=S_{16}+48 S_{7,7,2}-314 S_{6,6,4}, \\
S_{11,5}=-S_{16}-55 S_{7,7,2}+330 S_{6,6,4}, \\
S_{10,6}=S_{16}+60 S_{7,7,2}-335 S_{6,6,4}, \\
S_{9,7}=-S_{16}-63 S_{7,7,2}+336 S_{6,6,4}, \\
S_{8,8}=\frac{S_{16}+64 S_{7,7,2}-336 S_{6,6,4}}{2} .
\end{gathered}
$$

4) Solution of Constraints of degree 18:

The invariant polynomials $S_{18}, S_{14,2,2}, S_{7,7,4}, S_{8,5,5}$ are considered to be independent and the other dependent invariant polynomials can be written in terms of them as follows:

$$
\begin{gathered}
S_{7,6,5}=-S_{8,5,5}, \quad S_{6,6,6}=\frac{S_{8,5,5}}{3}, \\
S_{8,7,3}=-S_{7,7,4}, \quad S_{8,6,4}=S_{8,5,5}-2 S_{7,7,4}, \\
S_{9,5,4}=-3 S_{8,5,5}+2 S_{7,7,4}, \quad S_{9,6,3}=3 S_{7,7,4}-S_{8,5,5}, \\
S_{10,5,3}=4 S_{8,5,5}-5 S_{7,7,4}, \quad S_{10,4,4}=3 S_{8,5,5}-2 S_{7,7,4}, \\
S_{11,4,3}=9 S_{7,7,4}-10 S_{8,5,5}, \quad S_{12,3,3}=10 S_{8,5,5}-9 S_{7,7,4},
\end{gathered}
$$




$$
\begin{aligned}
& S_{13,3,2}=-S_{14,2,2}, \\
& S_{12,4,2}=S_{14,2,2}-20 S_{8,5,5}+18 S_{7,7,4} \\
& S_{11,5,2}=-S_{14,2,2}+30 S_{8,5,5}-27 S_{7,7,4}, \\
& S_{10,6,2}=32 S_{7,7,4}-34 S_{8,5,5}+S_{14,2,2}, \\
& S_{9,7,2}=35 S_{8,5,5}-35 S_{7,7,4}-S_{14,2,2}, \\
& S_{8,8,2}=\frac{36 S_{7,7,4}+S_{14,2,2}-35 S_{8,5,5}}{2}, \\
& S_{9,8,1}=\frac{35 S_{8,5,5}-S_{14,2,2}-36 S_{7,7,4}}{2}, \\
& S_{10,7,1}=\frac{106 S_{7,7,4}-105 S_{8,5,5}+3 S_{14,2,2}}{2}, \\
& S_{11,6,1}=\frac{173 S_{8,5,5}-170 S_{7,7,4}-5 S_{14,2,2}}{2}, \\
& S_{12,5,1}=\frac{224 S_{7,7,4}-233 S_{8,5,5}+7 S_{14,2,2}}{2}, \\
& S_{13,4,1}=\frac{273 S_{8,5,5}-260 S_{7,7,4}-9 S_{14,2,2}}{2}, \\
& S_{14,3,1}=260 S_{7,7,4}-273 S_{8,5,5}+11 S_{14,2,2}, \\
& S_{15,2,1}=\frac{273 S_{8,5,5}-260 S_{7,7,4}-15 S_{14,2,2}}{2}, \\
& S_{16,1,1}=\frac{260 S_{7,7,4}+15 S_{14,2,2}-273 S_{8,5,5}}{2}, \\
& S_{17,1}=-S_{18}, \\
& S_{16,2}=S_{18}-260 S_{7,7,4}-15 S_{14,2,2}+273 S_{8,5,5}, \\
& S_{15,3}=\frac{780 S_{7,7,4}-2 S_{18}+45 S_{14,2,2}-819 S_{8,5,5}}{2}, \\
& S_{14,4}=\frac{1092 S_{8,5,5}-56 S_{14,2,2}+2 S_{18}-1040 S_{7,7,4}}{2}, \\
& S_{13,5}=\frac{65 S_{14,2,2}-2 S_{18}-1365 S_{8,5,5}+1300 S_{7,7,4}}{2}, \\
& S_{12,6}=\frac{2 S_{18}+1598 S_{8,5,5}-1524 S_{7,7,4}-72 S_{14,2,2}}{2},
\end{aligned}
$$




$$
\begin{aligned}
S_{11,7} & =\frac{1694 S_{7,7,4}+77 S_{14,2,2}-2 S_{18}-1771 S_{8,5,5}}{2}, \\
S_{10,8} & =\frac{2 S_{18}+1876 S_{8,5,5}-80 S_{14,2,2}-1800 S_{7,7,4}}{2} \\
S_{9,9} & =\frac{81 S_{14,2,2}+1836 S_{7,7,4}-2 S_{18}-1911 S_{8,5,5}}{4} .
\end{aligned}
$$

5) Solution of Constraints of degree 20:

The invariant polynomials $S_{20}, S_{16,2,2}, S_{8,8,4}$ and $S_{7,7,6}$ are considered to be independent and the other dependent invariant polynomials can be written in terms of them as follows:

$$
\begin{gathered}
S_{8,8,6}=-2 S_{7,7,6}, \quad S_{8,7,5}=-S_{7,7,6}, \\
S_{9,6,5}=5 S_{7,7,6}, \quad S_{9,8,3}=-S_{8,8,4}, \\
S_{9,7,4}=S_{7,7,6}-2 S_{8,8,4}, \quad S_{10,5,5}=-5 S_{7,7,6}, \\
S_{10,6,4}=-6 S_{7,7,6}+2 S_{8,8,4}, \quad S_{10,7,3}=3 S_{8,8,4}-S_{7,7,6}, \\
S_{11,6,3}=-5 S_{8,8,4}+7 S_{7,7,6}, \quad S_{11,5,4}=+16 S_{7,7,6}-2 S_{8,8,4}, \\
S_{12,4,4}=\left(-16 S_{7,7,6}+2 S_{8,8,4}\right), \quad S_{12,5,3}=-23 S_{7,7,6}+7 S_{8,8,4}, \\
S_{13,4,3}=55 S_{7,7,6}-11 S_{8,8,4}, \quad S_{14,3,3}=-55 S_{7,7,6}+11 S_{8,8,4}, \\
S_{14,4,2}=-22 S_{8,8,4}+S_{16,2,2}+110 S_{7,7,6}, \\
S_{13,5,2}=-165 S_{7,7,6}-S_{16,2,2}+33 S_{8,8,4}, \\
S_{12,6,2}=-40 S_{8,8,4}+188 S_{7,7,6}+S_{16,2,2}, \\
S_{11,7,2}=-195 S_{7,7,6}+45 S_{8,8,4}-S_{16,2,2}, \\
S_{10,8,2}=-48 S_{8,8,4}+196 S_{7,7,6}+S_{16,2,2}, \\
S_{9,9,2}=\frac{-196 S_{7,7,6}+49 S_{8,8,4}-S_{16,2,2}}{2} \\
S_{10,9,1}=\frac{S_{16,2,2}+196 S_{7,7,6}-49 S_{8,8,4}}{2},
\end{gathered}
$$




$$
\begin{gathered}
S_{11,8,1}=\frac{-588 S_{7,7,6}+145 S_{8,8,4}-3 S_{16,2,2}}{2}, \\
S_{12,7,1}=\frac{+978 S_{7,7,6}-235 S_{8,8,4}+5 S_{16,2,2}}{2}, \\
S_{13,6,1}=\frac{-1354 S_{7,7,6}+315 S_{8,8,4}-7 S_{16,2,2}}{2}, \\
S_{14,5,1}=\frac{9 S_{16,2,2}-381 S_{8,8,4}+1684 S_{7,7,6}}{2}, \\
S_{15,4,1}=\frac{425 S_{8,8,4}-1904 S_{7,7,6}-11 S_{16,2,2}}{2}, \\
S_{16,3,1}=\frac{13 S_{16,2,2}+1904 S_{7,7,6}-425 S_{8,8,4}}{2}, \\
S_{17,2,1}=\frac{-1904 S_{7,7,6}+425 S_{8,8,4}-17 S_{16,2,2}}{2}, \\
S_{18,1,1}=\frac{17 S_{16,2,2}-425 S_{8,8,4}+1904 S_{7,7,6}}{2}, \\
S_{18,2}=S_{20}+425 S_{8,8,4}-1904 S_{7,7,6}-17 S_{16,2,2}, \\
S_{17,3}= \\
S_{16,4}=\frac{51 S_{16,2,2}-1275 S_{8,8,4}+5712 S_{7,7,6}-2 S_{20}}{2}, \\
S_{11,8}=\frac{2 S_{20}-64 S_{16,2,2}+1700 S_{8,8,4}-7616 S_{7,7,6}}{2}, \\
S_{15,5}=\frac{75 S_{16,2,2}-2125 S_{8,8,4}-2 S_{20}+9520 S_{7,7,6}}{2}, \\
S_{14,6}=\frac{2 S_{20}+2506 S_{8,8,4}-11204 S_{7,7,6}-84 S_{16,2,2}}{2}, \\
S_{13,7}=\frac{91 S_{16,2,2}+12558 S_{7,7,6}-2821 S_{8,8,4}-2 S_{20}}{2}, \\
2 S_{16,2,2}+3056 S_{8,8,4}-13536 S_{7,7,6} \\
2
\end{gathered}
$$

6) Solution of Constraints of degree 22: 
The invariant polynomials $S_{22}, S_{18,2,2}, S_{9,9,4}$ and $S_{8,8,6}$ are considered to be independent and the other dependent invariant polynomials can be written in terms of them as follows:

$$
\begin{aligned}
& S_{8,7,7}=0, \quad S_{9,7,6}=-2 S_{8,8,6}, \\
& S_{9,8,5}=-S_{8,8,6}, \quad S_{10,6,6}=2 S_{8,8,6}, \\
& S_{10,7,5}=3 S_{8,8,6}, \quad S_{10,9,3}=-S_{9,9,4}, \\
& S_{10,8,4}=S_{8,8,6}-2 S_{9,9,4}, \quad S_{11,6,5}=-7 S_{8,8,6}, \\
& S_{11,7,4}=2 S_{9,9,4}-4 S_{8,8,6}, \\
& S_{11,8,3}=3 S_{9,9,4}-S_{8,8,6}, \quad S_{12,5,5}=7 S_{8,8,6} \\
& S_{12,6,4}=11 S_{8,8,6}+2 S_{9,9,4}, \quad S_{12,7,3}=5 S_{8,8,6}-5 S_{9,9,4}, \\
& S_{13,5,4}=2 S_{9,9,4}-25 S_{8,8,6}, \quad S_{13,6,3}=7 S_{9,9,4}-16 S_{8,8,6}, \\
& S_{14,4,4}=25 S_{8,8,6}-2 S_{9,9,4}, \quad S_{14,5,3}=41 S_{8,8,6}-9 S_{9,9,4}, \\
& S_{15,4,3}=13 S_{9,9,4}-91 S_{8,8,6}, \quad S_{16,3,3}=91 S_{8,8,6}-13 S_{9,9,4}, \\
& S_{17,3,2}=-S_{18,2,2}, \\
& S_{16,4,2}=26 S_{9,9,4}-182 S_{8,8,6}+S_{18,2,2}, \\
& S_{15,5,2}=273 S_{8,8,6}-39 S_{9,9,4}-S_{18,2,2}, \\
& S_{14,6,2}=48 S_{9,9,4}-314 S_{8,8,6}+S_{18,2,2}, \\
& S_{13,7,2}=330 S_{8,8,6}-55 S_{9,9,4}-S_{18,2,2}, \\
& S_{12,8,2}=60 S_{9,9,4}+S_{18,2,2}-335 S_{8,8,6}, \\
& S_{11,9,2}=336 S_{8,8,6}-63 S_{9,9,4}-S_{18,2,2}, \\
& S_{10,10,2}=\frac{S_{18,2,2}+64 S_{9,9,4}-336 S_{8,8,6}}{2}, \\
& S_{11,10,1}=\frac{-64 S_{9,9,4}+336 S_{8,8,6}-S_{18,2,2}}{2},
\end{aligned}
$$




$$
\begin{aligned}
& S_{12,9,1}=\frac{190 S_{9,9,4}+3 S_{18,2,2}-1008 S_{8,8,6}}{2}, \\
& S_{13,8,1}=\frac{-310 S_{9,9,4}-5 S_{18,2,2}+1678 S_{8,8,6}}{2}, \\
& S_{14,7,1}=\frac{-2338 S_{8,8,6}+7 S_{18,2,2}+420 S_{9,9,4}}{2}, \\
& S_{15,6,1}=\frac{2966 S_{8,8,6}-9 S_{18,2,2}-516 S_{9,9,4}}{2}, \\
& S_{16,5,1}=\frac{11 S_{18,2,2}+594 S_{9,9,4}-3512 S_{8,8,6}}{2}, \\
& S_{17,4,1}=\frac{3876 S_{8,8,6}-13 S_{18,2,2}-646 S_{9,9,4}}{2}, \\
& S_{18,3,1}=\frac{-3876 S_{8,8,6}+646 S_{9,9,4}+15 S_{18,2,2}}{2}, \\
& S_{19,2,1}=\frac{-646 S_{9,9,4}-19 S_{18,2,2}+3876 S_{8,8,6}}{2}, \\
& S_{20,1,1}=\frac{-3876 S_{8,8,6}+19 S_{18,2,2}+646 S_{9,9,4}}{2}, \\
& S_{21,1}=-S_{22}, \\
& S_{20,2}=S_{22}-19 S_{18,2,2}-646 S_{9,9,4}+3876 S_{8,8,6}, \\
& S_{19,3}=\frac{57 S_{18,2,2}-2 S_{22}+1938 S_{9,9,4}-11628 S_{8,8,6}}{2}, \\
& S_{18,4}=\frac{2 S_{22}-72 S_{18,2,2}-2584 S_{9,9,4}+15504 S_{8,8,6}}{2}, \\
& S_{17,5}=\frac{3230 S_{9,9,4}+85 S_{18,2,2}-19380 S_{8,8,6}-2 S_{22}}{2}, \\
& S_{16,6}=\frac{2 S_{22}-96 S_{18,2,2}-3824 S_{9,9,4}+22892 S_{8,8,6}}{2}, \\
& S_{15,7}=\frac{105 S_{18,2,2}+4340 S_{9,9,4}-25858 S_{8,8,6}-2 S_{22}}{2}, \\
& S_{14,8}=\frac{28196 S_{8,8,6}-112 S_{18,2,2}+2 S_{22}-4760 S_{9,9,4}}{2}, \\
& S_{13,9}=\frac{5070 S_{9,9,4}+117 S_{18,2,2}-29874 S_{8,8,6}-2 S_{22}}{2}, \\
& S_{12,10}=\frac{30882 S_{8,8,6}+2 S_{22}-120 S_{18,2,2}-5260 S_{9,9,4}}{2}, \\
& S_{11,11}=\frac{121 S_{18,2,2}+4678 S_{9,9,4}-2 S_{22}-31218 S_{8,8,6}}{4} .
\end{aligned}
$$


APPENDIX IV:the recursion relation and $\lambda_{\max }$ for $q \geq 6$ a) $\mathbf{b}=\mathbf{2} \mathbf{q}=\mathbf{6}$

$$
\begin{aligned}
\left(\begin{array}{c}
A_{6(n+1)} \\
A_{4,1,1(n+1)}
\end{array}\right) & =\left(\begin{array}{cc}
1.090534979 & -.1646090535 \\
.1481481481 & .1851851852
\end{array}\right)\left(\begin{array}{c}
A_{6(n)} \\
A_{4,1,1(n)}
\end{array}\right), \\
\lambda_{\max } & =1.062745991 .
\end{aligned}
$$

$q=8$

$$
\left(\begin{array}{c}
A_{8(n+1)} \\
A_{3,3,2(n+1)}
\end{array}\right)=\left(\begin{array}{cc}
1.039323274 & .5121170553 \\
-.03840877915 & .1742112483
\end{array}\right)\left(\begin{array}{c}
A_{8(n)} \\
A_{3,3,2(n)}
\end{array}\right),
$$$$
\lambda_{\max }=1.015955376 .
$$

$q=10$

$$
\left(\begin{array}{c}
A_{10(n+1)} \\
A_{4,4,2(n+1)}
\end{array}\right)=\left(\begin{array}{cc}
1.017375400 & -.3840877915 \\
.02987349489 & .1486053955
\end{array}\right)\left(\begin{array}{c}
A_{10(n)} \\
A_{4,4,2(n)}
\end{array}\right),
$$

$$
\lambda_{\max }=1.003961045 .
$$

$q=12$

$$
\begin{aligned}
\left(\begin{array}{c}
A_{12(n+1)} \\
A_{8,2,2(n+1)} \\
A_{6,3,3(n+1)}
\end{array}\right)= & \left(\begin{array}{ccc}
1.007711110 & -.1266744568 & .1217068310 \\
.05378583888 & .1908753747 & -.1417805551 \\
.007315957933 & .07681755830 & -.04709647920
\end{array}\right)\left(\begin{array}{c}
A_{12(n)} \\
A_{8,2,2(n)} \\
A_{6,3,3(n+1)}
\end{array}\right), \\
& \lambda_{\max }=1.000983621 .
\end{aligned}
$$


$\mathrm{q}=14$

$$
\left(\begin{array}{c}
A_{14(n+1)} \\
A_{6,6,2(n+1)} \\
A_{5,5,4(n+1)}
\end{array}\right)=\left(\begin{array}{ccc}
1.003425906 & -.1557074696 & .6579896295 \\
.01048470103 & -.09541040304 & .5668362057 \\
-.001896729835 & -.04335382479 & .2332808346
\end{array}\right)\left(\begin{array}{c}
A_{14(n)} \\
A_{6,6,2(n)} \\
A_{5,5,4(n+1)}
\end{array}\right)
$$

$$
\lambda_{\max }=1.000244974 .
$$

$q=16$

$$
\left(\begin{array}{c}
A_{16(n+1)} \\
A_{7,7,2(n+1)} \\
A_{6,6,4(n+1)}
\end{array}\right)=\left(\begin{array}{ccc}
1.001522485 & .09132402907 & -.5053262942 \\
-.006679533152 & -.1334905160 & 1.056414123 \\
.001475234316 & -.03567658498 & .2661499583
\end{array}\right)\left(\begin{array}{c}
A_{16(n)} \\
A_{7,7,2(n)} \\
A_{6,6,4(n+1)}
\end{array}\right),
$$

$$
\lambda_{\max }=1.000061131 .
$$

$$
\mathrm{q}=18
$$

$$
\left(\begin{array}{c}
A_{18(n+1)} \\
A_{14,2,2(n+1)} \\
A_{7,7,4(n+1)} \\
A_{8,5,5(n+1)}
\end{array}\right)=\left(\begin{array}{cccc}
1.000676645 & -.02587988061 & -.5694995445 & .5946011699 \\
.04976341868 & .3702685508 & 6.165437409 & -6.474053436 \\
-.0007861225946 & -.004847198466 & -.1026962541 & .1087868226 \\
.0003612818732 & .009483649173 & .1320936849 & -.1378233896 \\
1.000015270 & .1122123554 & .01717707276 & .001020854222
\end{array}\right)\left(\begin{array}{c}
A_{18(n)} \\
A_{14,2,2(n)} \\
A_{7,7,4(n)} \\
A_{8,5,5(n)}
\end{array}\right),
$$

$$
\lambda_{\max }=1.000015270 .
$$

$\mathrm{q}=20$

$$
\left(\begin{array}{c}
A_{20(n+1)} \\
A_{16,2,2(n+1)} \\
A_{8,8,4(n+1)} \\
A_{7,7,6(n+1)}
\end{array}\right)=\left(\begin{array}{cccc}
1.000300729 & -.01428439338 & .4535545013 & -2.007507524 \\
.04955188625 & .4249966010 & -10.27981253 & 46.05861448 \\
.0005177630138 & .0009212316079 & -.05329922806 & .2455943625 \\
-.00009366567084 & -.002585841556 & .05473587640 & -.2437584270
\end{array}\right)\left(\begin{array}{c}
A_{20(n)} \\
A_{16,2,2(n)} \\
A_{8,8,4(n)} \\
A_{7,7,6(n)}
\end{array}\right),
$$




$$
\lambda_{\max }=1.000003815 .
$$

$\mathrm{q}=22$

$$
\left(\begin{array}{c}
A_{22(n+1)} \\
A_{18,2,2(n+1)} \\
A_{9,9,4(n+1)} \\
A_{8,8,6(n+1)}
\end{array}\right)=\left(\begin{array}{cccc}
1.000133657 & -.007718672824 & -.3331935728 & 1.963553834 \\
.04945789936 & .4775535736 & 15.78748967 & -94.73506749 \\
-.0003298534890 & .001784479705 & .02202297308 & -.1217001716 \\
.00007285107732 & .002090751581 & .06241497465 & -.3728127167
\end{array}\right)\left(\begin{array}{c}
A_{22(n)} \\
A_{18,2,2(n)} \\
A_{9,9,4(n)} \\
A_{8,8,6(n)}
\end{array}\right),
$$

$$
\lambda_{\max }=1.000000954 \text {. }
$$

b) $b=3 q=6$

$$
\begin{aligned}
\left(\begin{array}{c}
A_{6(n+1)} \\
A_{4,1,1(n+1)}
\end{array}\right) & =\left(\begin{array}{cc}
1.082584637 & -.07343878826 \\
.1808430161 & .04755671531
\end{array}\right)\left(\begin{array}{c}
A_{6(n)} \\
A_{4,1,1(n)}
\end{array}\right), \\
\lambda_{\max } & =1.069590059 .
\end{aligned}
$$

$q=8$

$$
\begin{aligned}
\left(\begin{array}{c}
A_{8(n+1)} \\
A_{3,3,2(n+1)}
\end{array}\right) & =\left(\begin{array}{cc}
1.025056713 & .1818484281 \\
-.04533096632 & .03647844913
\end{array}\right)\left(\begin{array}{c}
A_{8(n)} \\
A_{3,3,2(n)}
\end{array}\right) \\
\lambda_{\max } & =1.016646559 .
\end{aligned}
$$

$q=10$

$$
\left(\begin{array}{c}
A_{10(n+1)} \\
A_{4,4,2(n+1)}
\end{array}\right)=\left(\begin{array}{cc}
1.007845912 & -.1085523780 \\
.03420274178 & .02815706873
\end{array}\right)\left(\begin{array}{c}
A_{10(n)} \\
A_{4,4,2(n)}
\end{array}\right)
$$




$$
\lambda_{\max }=1.004041374 .
$$

$q=12$

$$
\left(\begin{array}{c}
A_{12(n+1)} \\
A_{8,2,2(n+1)} \\
A_{6,3,3(n+1)}
\end{array}\right)=\left(\begin{array}{ccc}
1.002501314 & -.02774717658 & .02388825224 \\
.06022405661 & .05660671065 & -.04390015579 \\
.01079114607 & .01664662055 & -.01252511118
\end{array}\right)\left(\begin{array}{c}
A_{12(n)} \\
A_{8,2,2(n)} \\
A_{6,3,3(n+1)}
\end{array}\right),
$$

$$
\lambda_{\max }=1.001026135 .
$$

$q=14$

$$
\left(\begin{array}{c}
A_{14(n+1)} \\
A_{6,6,2(n+1)} \\
A_{5,5,4(n+1)}
\end{array}\right)=\left(\begin{array}{ccc}
1.000805713 & -.02587192638 & .1198110012 \\
.008633787717 & -.02491545222 & .1279091010 \\
-.002716116832 & -.009331353906 & .04723947173
\end{array}\right)\left(\begin{array}{c}
A_{14(n)} \\
A_{6,6,2(n)} \\
A_{5,5,4(n+1)}
\end{array}\right),
$$

$$
\lambda_{\max }=1.000246359 .
$$

$$
q=16
$$

$$
\left(\begin{array}{c}
A_{16(n+1)} \\
A_{7,7,2(n+1)} \\
A_{6,6,4(n+1)}
\end{array}\right)=\left(\begin{array}{ccc}
1.000261069 & .01135511506 & -.07104143816 \\
-.004468408282 & -.03190697902 & .2276301973 \\
.002050937173 & -.007546589748 & .05339121346
\end{array}\right)\left(\begin{array}{c}
A_{16(n)} \\
A_{7,7,2(n)} \\
A_{6,6,4(n+1)}
\end{array}\right),
$$

$$
\lambda_{\max }=1.000061317 .
$$


$\mathrm{q}=18$

$$
\left(\begin{array}{c}
A_{18(n+1)} \\
A_{14,2,2(n+1)} \\
A_{7,7,4(n+1)} \\
A_{8,5,5(n+1)}
\end{array}\right)=\left(\begin{array}{cccc}
1.000084877 & -.002388883753 & -.04740112573 & .04969629320 \\
.06005002155 & .07399773075 & 1.274170117 & -1.337882220 \\
-.0009014749149 & -.0004616094519 & -.008788472030 & .009233751643 \\
.0006471907605 & .002494396769 & .04199894504 & -.04409378714
\end{array}\right)\left(\begin{array}{c}
A_{18(n)} \\
A_{1422(n)} \\
A_{7,7,4(n)} \\
A_{8,5,5(n)}
\end{array}\right),
$$

$$
\lambda_{\max }=1.000015297 .
$$

$\mathrm{q}=20$

$$
\left(\begin{array}{c}
A_{20(n+1)} \\
A_{16,2,2(n+1)} \\
A_{8,8,4(n+1)} \\
A_{7,7,6(n+1)}
\end{array}\right)=\left(\begin{array}{cccc}
1.000027647 & -.0009744218073 & .02806977909 & -.1253211808 \\
.05999917318 & .08477443355 & -2.107893057 & 9.443400292 \\
.0005178070963 & -.0004282216637 & .009448647802 & -.04228755067 \\
-.0001628983371 & -.0006696634259 & .01635654929 & -.07326845888
\end{array}\right)\left(\begin{array}{c}
A_{20(n)} \\
A_{16,2,2(n)} \\
A_{8,8,4(n)} \\
A_{7,7,6(n)}
\end{array}\right),
$$

$$
\lambda_{\max }=1.000003819 .
$$

$\mathrm{q}=22$

$$
\left(\begin{array}{c}
A_{22(n+1)} \\
A_{18,2,2(n+1)} \\
A_{9,9,4(n+1)} \\
A_{8,8,6(n+1)}
\end{array}\right)=\left(\begin{array}{cccc}
1.000006761 & -.0002279253840 & -.009315026611 & .05551458536 \\
.05998283956 & .09512343412 & 3.219304290 & -19.31589634 \\
-.0002679923170 & .0009873502040 & .03199666409 & -.1919144288 \\
.0001230048643 & .0005306869789 & .01770755556 & -.1062348858
\end{array}\right)\left(\begin{array}{c}
A_{22(n)} \\
A_{18,2,2(n)} \\
A_{9,9,4(n)} \\
A_{8,8,6(n)}
\end{array}\right),
$$

$$
\lambda_{\max }=1.000002379 .
$$

c) $b=4 q=6$

$$
\begin{aligned}
\left(\begin{array}{c}
A_{6(n+1)} \\
A_{4,1,1(n+1)}
\end{array}\right) & =\left(\begin{array}{cc}
1.076310660 & -.03365228885 \\
.1889456076 & .01616947736
\end{array}\right)\left(\begin{array}{c}
A_{6(n)} \\
A_{4,1,1(n)}
\end{array}\right) \\
\lambda_{\max } & =1.070278597
\end{aligned}
$$




$$
\begin{gathered}
\mathbf{q}=8 \\
\left(\begin{array}{c}
A_{8(n+1)} \\
A_{3,3,2(n+1)}
\end{array}\right)=\left(\begin{array}{cc}
1.019524120 & .06535850011 \\
-.05434716964 & 371.8425102
\end{array}\right)\left(\begin{array}{c}
A_{8(n)} \\
A_{3,3,2(n)}
\end{array}\right), \\
\lambda_{\max }=1.0195338 .
\end{gathered}
$$

$q=10$

$$
\left(\begin{array}{c}
A_{10(n+1)} \\
A_{4,4,2(n+1)}
\end{array}\right)=\left(\begin{array}{cc}
1.005151073 & -.03282144763 \\
.03495279823 & .007384841623
\end{array}\right)\left(\begin{array}{c}
A_{10(n)} \\
A_{4,4,2(n)}
\end{array}\right),
$$

$$
\lambda_{\max }=1.003999975 .
$$

$$
\mathrm{q}=12
$$$$
\left(\begin{array}{c}
A_{12(n+1)} \\
A_{8,2,2(n+1)} \\
A_{6,3,3(n+1)}
\end{array}\right)=\left(\begin{array}{ccc}
1.001388320 & -.007332723661 & .006026327075 \\
.06394351082 & .009657753638 & -.007508055989 \\
.01147176558 & .004507477340 & -.003470225654
\end{array}\right)\left(\begin{array}{c}
A_{12(n)} \\
A_{8,2,2(n)} \\
A_{6,3,3(n+1)}
\end{array}\right)
$$

$$
\lambda_{\max }=1.000986557 \text {. }
$$

$$
\mathrm{q}=14
$$

$$
\left(\begin{array}{c}
A_{14(n+1)} \\
A_{6,6,2(n+1)} \\
A_{5,5,4(n+1)}
\end{array}\right)=\left(\begin{array}{ccc}
1.000380234 & -.006079338806 & .02938006638 \\
.008215782561 & -.006916822481 & .03482160520 \\
-.002872694778 & -.002513285925 & .01260695484
\end{array}\right)\left(\begin{array}{c}
A_{14(n)} \\
A_{6,6,2(n)} \\
A_{5,5,4(n+1)}
\end{array}\right),
$$




$$
\lambda_{\max }=1.000245203 .
$$

\section{$q=16$}

$$
\left(\begin{array}{c}
A_{16(n+1)} \\
A_{7,7,2(n+1)} \\
A_{6,6,4(n+1)}
\end{array}\right)=\left(\begin{array}{ccc}
1.000105428 & .002387138955 & -.01597611501 \\
-.004013610557 & -.008712843003 & .06128427304 \\
.002158295483 & -.002024493055 & .01421013434
\end{array}\right)\left(\begin{array}{c}
A_{16(n)} \\
A_{7,7,2(n)} \\
A_{6,6,4(n+1)}
\end{array}\right),
$$

$$
\lambda_{\max }=1.000061148 .
$$

$\mathrm{q}=18$

$$
\left(\begin{array}{c}
A_{18(n+1)} \\
A_{14,2,2(n+1)} \\
A_{7,7,4(n+1)} \\
A_{8,5,5(n+1)}
\end{array}\right)=\left(\begin{array}{cccc}
1.000029509 & -.0004497502350 & -.008243519261 & .008652383249 \\
.06187422841 & .01967111602 & .3403871980 & -.3574066079 \\
-.0009123885980 & -.00009719487958 & -.001741086261 & .001828250148 \\
.0007092075724 & .0006921244620 & .01190983853 & -.01250523720
\end{array}\right)\left(\begin{array}{c}
A_{18(n)} \\
A_{14,2,2(n)} \\
A_{7,7,4(n)} \\
A_{8,5,5(n)}
\end{array}\right),
$$

$$
\lambda_{\max }=1.000015271 .
$$

\section{$\mathrm{q}=\mathbf{2 0}$}

$$
\left(\begin{array}{c}
A_{20(n+1)} \\
A_{16,2,2(n+1)} \\
A_{8,8,4(n+1)} \\
A_{7,7,6(n+1)}
\end{array}\right)=\left(\begin{array}{cccc}
1.000008319 & -.0001645061367 & .004348107103 & -.01946561802 \\
.06184409730 & .02252802695 & -.5624149410 & 2.519619600 \\
.0005078921879 & -.0001439100811 & .003508323524 & -.01571652660 \\
-.0001776181030 & -.0001853563027 & .004606804705 & -.02063832629
\end{array}\right)\left(\begin{array}{c}
A_{20(n)} \\
A_{16,2,2(n)} \\
A_{8,8,4(n)} \\
A_{7,7,6(n)}
\end{array}\right),
$$

$$
\lambda_{\max }=1.000003788 .
$$

\section{$\mathrm{q}=22$}

$$
\left(\begin{array}{c}
A_{22(n+1)} \\
A_{18,2,2(n+1)} \\
A_{9,9,4(n+1)} \\
A_{8,8,6(n+1)}
\end{array}\right)=\left(\begin{array}{cccc}
1.000002358 & -.00005834048617 & -.002108427732 & .01263785041 \\
.06183577589 & .02527262136 & .8582479458 & -5.149488875 \\
-.0002481471736 & .0002926869789 & .009839879848 & -.05903810198 \\
.0001334512767 & .0001464026161 & .004954070521 & -.02972423554
\end{array}\right)\left(\begin{array}{c}
A_{22(n)} \\
A_{18,2,2(n)} \\
A_{9,9,4(n)} \\
A_{8,8,6(n)}
\end{array}\right),
$$




$$
\lambda_{\max }=1.000000954 .
$$

d) $b=5 \quad q=6$

$$
\left(\begin{array}{c}
A_{6(n+1)} \\
A_{4,1,1(n+1)}
\end{array}\right)=\left(\begin{array}{cc}
1.044360741 & -.01006023599 \\
.3156061304 & .003157528970
\end{array}\right)\left(\begin{array}{c}
A_{6(n)} \\
A_{4,1,1(n)}
\end{array}\right),
$$

$$
\lambda_{\max }=1.041302331 .
$$

$q=8$

$$
\left(\begin{array}{c}
A_{8(n+1)} \\
A_{3,3,2(n+1)}
\end{array}\right)=\left(\begin{array}{cc}
1.009037038 & .01421998195 \\
.07691145227 & -.005139564359
\end{array}\right)\left(\begin{array}{c}
A_{8(n)} \\
A_{3,3,2(n)}
\end{array}\right),
$$

$$
\lambda_{\max }=1.010114286 .
$$

$q=10$

$$
\left(\begin{array}{c}
A_{10(n+1)} \\
A_{4,4,2(n+1)}
\end{array}\right)=\left(\begin{array}{cc}
1.001897065 & -.005511338227 \\
.03479355808 & .002253185745
\end{array}\right)\left(\begin{array}{c}
A_{10(n)} \\
A_{4,4,2(n)}
\end{array}\right),
$$

$$
\lambda_{\max }=1.001705201 .
$$

\section{$q=12$}

$$
\left(\begin{array}{c}
A_{12(n+1)} \\
A_{8,2,2(n+1)} \\
A_{6,3,3(n+1)}
\end{array}\right)=\left(\begin{array}{ccc}
1.000405841 & -.0009777920845 & .0007888947248 \\
.06389106764 & .002988237303 & -.002324086713 \\
.01925523989 & .001402362796 & -.001089147707
\end{array}\right)\left(\begin{array}{c}
A_{12(n)} \\
A_{8,2,2(n)} \\
A_{6,3,3(n+1)}
\end{array}\right),
$$




$$
\lambda_{\max }=1.000358489 .
$$

$$
\begin{gathered}
\mathbf{q = 1 4} \\
\left(\begin{array}{c}
A_{14(n+1)} \\
A_{6,6,2(n+1)} \\
A_{5,5,4(n+1)}
\end{array}\right)=\left(\begin{array}{ccc}
1.000087998 & -.0006533768126 & .003203625771 \\
.008004412267 & -.002154118594 & .01079323997 \\
.004778768070 & .001472733806 & -.007372115949
\end{array}\right)\left(\begin{array}{c}
A_{14(n)} \\
A_{6,6,2(n)} \\
A_{5,5,4(n+1)}
\end{array}\right), \\
\lambda_{\max }=1.000097981 .
\end{gathered}
$$

\section{$\mathrm{q}=16$}

$$
\left(\begin{array}{c}
A_{16(n+1)} \\
A_{7,7,2(n+1)} \\
A_{6,6,4(n+1)}
\end{array}\right)=\left(\begin{array}{ccc}
1.000019275 & .0002082580899 & -.001424787973 \\
.003961084604 & .002614693987 & -.01832747999 \\
.002150223007 & -.0006222811611 & .004359625253
\end{array}\right)\left(\begin{array}{c}
A_{16(n)} \\
A_{7,7,2(n)} \\
A_{6,6,4(n+1)}
\end{array}\right),
$$

$$
\lambda_{\max }=1.000017019 \text {. }
$$

\section{$q=18$}

$$
\begin{gathered}
\left(\begin{array}{c}
A_{18(n+1)} \\
A_{1422(n+1)} \\
A_{774(n+1)} \\
A_{855(n+1)}
\end{array}\right)=\left(\begin{array}{cccc}
1.000004256 & -.00003193265577 & -.0005685434366 & .0005968699293 \\
.06193405435 & .006096146153 & .1056112005 & -.1108917618 \\
.001017169377 & .00006930842110 & .001204750315 & -.001264989081 \\
.001191712555 & .0002627970450 & .004548661710 & -.004776093729
\end{array}\right)\left(\begin{array}{c}
A_{18(n)} \\
A_{14,2,2(n)} \\
A_{7,7,4(n)} \\
A_{855(n)}
\end{array}\right), \\
\lambda_{\max }=1.000002408 .
\end{gathered}
$$

\section{$\mathrm{q}=\mathbf{2 0}$}

$$
\left(\begin{array}{c}
A_{20(n+1)} \\
A_{16,2,2(n+1)} \\
A_{8,8,4(n+1)} \\
A_{7,7,6(n+1)}
\end{array}\right)=\left(\begin{array}{cccc}
1.000000946 & -.000009482285099 & .0002434259750 & -.001090262082 \\
.06190933065 & .006979673785 & -.1744166676 & .7813866910 \\
.0004953876882 & -.00004636993771 & .001150699865 & -.005155112958 \\
.0002958221549 & .0001041331110 & -.002597984312 & .01163895822
\end{array}\right)\left(\begin{array}{c}
A_{20(n)} \\
A_{16,2,2(n)} \\
A_{8,8,4(n)} \\
A_{7,7,6(n)}
\end{array}\right),
$$




$$
\lambda_{\max }=1.000000141 .
$$

$\mathrm{q}=22$

$$
\begin{gathered}
\left(\begin{array}{c}
A_{22(n+1)} \\
A_{18,2,2(n+1)} \\
A_{9,9,4(n+1)} \\
A_{8,8,6(n+1)}
\end{array}\right)=\left(\begin{array}{cccc}
1.000000211 & -.000002740626906 & -.00009573710813 & .0005742469550 \\
.06190294246 & .007829018982 & .2660888394 & -1.596533072 \\
.0002452104252 & -.00008750002217 & .2660888394 & .01778872116 \\
.0001330977107 & .00004502739760 & .001528690467 & -.009172137322
\end{array}\right)\left(\begin{array}{c}
A_{22(n)} \\
A_{18,2,2(n)} \\
A_{9,9,4(n)} \\
A_{8,8,6(n)}
\end{array}\right), \\
\lambda_{\max }=1.000000086 .
\end{gathered}
$$

\section{ACKNOWLEDGEMENT}

We wish to thank Dr. S. K. A. Seyed Yagoobi for his careful reading the article and for his constructive comments.

\section{References}

[1] B. B. Mandelbrot, The Fractal Geometry of Nature (Freeman, New York, 1982)

[2] M. Schroeder, Fractals, Chaos, Power laws, Minutes from an Infinite Paradise (Freeman, New York, 1991).

[3] Y. Gefen, A. Aharony, B. B. Mandelbrot and S. Kirkpatrich; Physics Review Letter 47 (1981) 1771.

[4] J. P. Clerc, G. Giraud, J. M. Laugier and J.M.Luck Advances in Physics, vol 39 no 3 (1990) 191.

[5] Stephane Roux and Catalin D. Mitescu, Physical review B 35 (1987) 898.

[6] Pak-Yee Tong and Kin-Wah Yu, Physics letters A 160 (1991) 293. 
[7] R. Julllian and R. Botet, Aggregation and Fractal Aggregates (World Scientific, Singapore, 1987).

[8] M.A. Jafarizadeh and S.K.A. Seyed-Yagoobi, Physics Letters A192 (1994) 109.

[9] M.A. Jafarizadeh and S.K.A. Seyed-Yagoobi, J. Polymer Science Part B: Polymer Physics 34, John Wiley \& Sons Inc. (1996)

[10] M.A. Jafarizadeh and S.K.A. Seyed-Yagoobi, Indian Journal of Physics 70 B 2 (1996) 751.

[11] M.A. Jafarizadeh and S.K.A. Seyed-Yagoobi, Europian Physical Journal B 7(1999) 429.

[12] M.A. Jafarizadeh, Europian Physical Journal B 4(1998) 103.

[13] M.A. Jafarizadeh, Restoration of macroscopic isotropy on d+1-simplex fractal conductors, cond-mat/000522\}(2000) to be published in Physica A.

[14] George E. Andrews, The Theory of Partitions (Addison-Wesley, London, 1976). 


\section{Figures Captions}

Figur 1. Sierpinsky fractal with decimation number $b=3$, prtitions of 2 denote the subfractals and partitions of 3 indicate the vertices, respectively.

Figure 2. Sierpinski fractal resistor networks with decimation number $b=3$. 
This figure "frac1.jpg" is available in "jpg" format from: http://arxiv.org/ps/cond-mat/0005338v1 
This figure "frac2.jpg" is available in "jpg" format from: http://arxiv.org/ps/cond-mat/0005338v1 